\title{
Enlightenment for Clinicians From the Case of Digestive Tract Metastases From Primary Lung Cancer: a Case Report
}

Shaochun Wang ( $\nabla$ wangshaochun9176@126.com)

Shanxi Provincial Peoples Hospital https://orcid.org/0000-0002-1409-5907

Shuili Wang

Shanxi Provincial Peoples Hospital

Wei Zhang

Shaanxi Provincial People's Hospital

Yeqin Zhang

Shaanxi Provincial People's Hospital

\section{Case report}

Keywords: Lung cancer, Digestive tract, Small intestinal metastases

Posted Date: October 11th, 2021

DOI: https://doi.org/10.21203/rs.3.rs-494539/v1

License: (c) (i) This work is licensed under a Creative Commons Attribution 4.0 International License. Read Full License 


\section{Abstract}

Background: About $50 \%$ of patients with initially diagnosed lung cancer have developed distant metastasis. The probability of occurrence of digestive tract metastases found by autopsy is rarely. This report presents a rare clinical case of small intestinal metastasis from primary squamous cell carcinoma of the lung.

Case presentation: An 82-year-old male with aggravated cough and more acute shortness of breath than usual was diagnosed with primary squamous cell carcinoma of the lung Stage IV, because of the advanced age, underlying diseases, and poor cardiopulmonary function,the family refused the chemoradiotherapy.

One month later, the patient gradually developed right lower quadrant pain. An upright abdominal X-ray suggested small intestinal obstruction and partial resection and anastomosis of the small intestine were performed. Postoperative pathology revealed poorly differentiated squamous-cell carcinoma of the small intestine. The patient died of sudden cardio-respiratory arrest one month later.

Conclusions: We conclude that the real incidence of digestive tract metastases from lung cancer may be higher than the reported because of the symptoms of those patients are not obvious. Clinicians should select the appropriate examination methods (such as positron emission tomography) and pay attention to the assessment of abdominal hollow organs.

\section{Background}

Bronchogenic carcinoma is the leading cause of death from malignant tumors [1], and $50 \%$ of patients with initially diagnosed lung cancer have developed distant metastasis [2]. The common sites of metastases from lung cancer are brain, liver, adrenal glands and bones [3]. The probability of occurrence of digestive tract metastases from primary bronchogenic carcinoma found by autopsy is $0.2-11.9 \%$ [4-5]. The average time from the diagnosis of gastrointestinal metastasis from lung cancer to death has been reported to be 130 days, suggesting a poor prognosis ${ }^{[2]}$. However, it has also been reported that a patient with small intestinal metastases from lung cancer who underwent surgical treatment for intestinal perforation in conjunction with surgical resection of the primary lung lesion survived 5 years after surgery with no significant disease progression. The researchers concluded that internists should be aware that patients with small intestinal metastases need to be treated surgically because the metastases are likely to lead to life-threatening complications such as small intestinal perforation. For some lung cancer patients, long-term survival can be achieved through resection and timely treatment of primary and metastatic lesions [6]. Therefore, patients can benefit from early detection of digestive tract metastases from lung cancer and appropriate intervention [7].

The patient, an 82-year-old male, had underlying "chronic obstructive pulmonary disease, chronic pulmonary heart disease, and coronary atherosclerotic heart disease". On September 5, 2018, the patient underwent a chest CT due to aggravated cough and more acute shortness of breath than usual, which 
revealed a mass-like soft tissue density shadow in the left upper lobe (neoplasms cannot be excluded), left pleural thickening, and left pleural effusion (Fig. A). Tumor marker test on admission revealed carcinoembryonic antigen $70.94 \mathrm{ng} / \mathrm{mL}$, neuron-specific enolase $21.72 \mathrm{ng} / \mathrm{mL}$, cytokeratin 19 fragment $14.5 \mathrm{ng} / \mathrm{mL}$, carbohydrate antigen CA-125 $104.4 \mathrm{U} / \mathrm{mL}$, and squamous cell carcinoma antigen 2.65 $\mathrm{ng} / \mathrm{mL}$. Bronchoscopy showed swelling and stenosis of the mucosa in the anterior segment of the left upper lobe, widening of the cristae, and a few nodular proliferations (Fig. B). Bronchoscopic biopsy pathology of the anterior segment of the left upper lobe showed moderately differentiated squamous cell carcinoma, immunohistochemistry: CK5/6 (+), P40 (+), p63 (+), CK7 (-), TTF-1 (-), NapsinA (-), and Ki-67 index $10 \%$ (Fig. C). The family refused further treatment because of the patient's advanced age, underlying diseases, and poor cardiopulmonary function. On September 28, 2018, the patient's shortness of breath worsened again, and a chest CT revealed that the amount of left pleural effusion was significantly increased compared to the previous test. The symptoms were significantly relieved after symptomatic treatment such as closed thoracic drainage and improvement of heart function. On October 4,2018 , the patient gradually developed right lower quadrant pain, which progressively worsened. An upright abdominal X-ray suggested small intestinal obstruction and an abdominal CT showed the small intestine was dilated and filled with fluid. The patient was treated with gastrointestinal decompression and temporary dietary abstinence, but the abdominal pain progressively worsened, and an emergency exploratory laparotomy was performed, which revealed a tumorous lesion in the small intestine about 50 $\mathrm{cm}$ from the ileocecal region, about $2 \mathrm{~cm} \times 3 \mathrm{~cm}$ in size, invading the whole thickness and causing local intestinal stenosis and adhesion to the small intestine about $20 \mathrm{~cm}$ away from its proximal end. The small intestine at the proximal end of the stenosis was dilated and filled with fluid, and the distal small intestine and colon were empty. Intraoperative diagnosis: small intestinal tumor with mechanical small intestinal obstruction. Partial resection and anastomosis of the small intestine was performed. Postoperative pathology revealed poorly differentiated carcinoma of the small intestine, which was squamous-cell carcinoma, tumor invading the entire thickness of the small intestinal wall, tumor thrombus in the vessels, and no cancer at the upper and lower ends (Fig. D). On November 5, 2018, the patient died of sudden cardio-respiratory arrest.

The currently reported incidence of digestive tract metastases from lung cancer is low, but its real incidence may be higher than the reported [8], and may even be much higher than the reported. A typical clinical symptoms of patients with digestive tract metastasis from lung cancer: There are many reports of small intestinal metastasis from lung cancer, which is mainly closely related to the fact that patients with small intestinal metastasis usually have obvious clinical symptoms (perforation, obstruction, bleeding) that are easy to detect, while patients with metastases to other parts of the body, such as the colon, often have insidious symptoms[9], which are easily mistaken for adverse digestive tract reactions after chemotherapy and other treatments, and are easily ignored. Therefore, clinicians should pay attention to gastrointestinal symptoms of lung cancer patients to avoid neglecting and missing the diagnosis of gastrointestinal metastases from lung cancer [10]. In particular, clinicians should be more vigilant when the fecal occult blood test is positive [11]. 
Low sensitivity of existing detection methods: Abdominal CT and other imaging examinations have low sensitivity in the early stage of detecting digestive tract metastasis, and there is no significant difference in the clinical symptoms between digestive tract metastasis from lung cancer and primary digestive tract tumors, so endoscopy cannot make a definite diagnosis, and histopathological examination must be relied on for differentiation [12]. For patients with initially diagnosed lung cancer, physicians will routinely arrange relevant examinations to assess whether there are metastases to the brain, bones, and abdominal parenchyma organs, but do not routinely assess metastases to abdominal hollow organs (digestive tract), which to some extent affects the diagnostic rate of digestive tract metastases from lung cancer.

In a patient with lung cancer, PET-CT showed enhanced local metabolism in the left upper abdomen at the time of initial diagnosis, however, neither abdominal CT nor digestive tract endoscopy revealed any abnormality, and digestive tract metastases were found within 3 months after the patient underwent resection of the primary lung lesion[9]. To a certain extent, this case tells us that the application of PET-CT plays an important role in the early diagnosis of digestive tract metastases from lung cancer, and especially for those patients with no clinical symptoms of digestive tract metastases from lung cancer, PET-CT can also make a definite diagnosis [13-14]. Therefore, in the systemic assessment of patients with initially diagnosed primary lung cancer, clinicians should select the appropriate examination methods and pay attention to the assessment of abdominal hollow organs.

The application of PET-CT plays an important role in the early diagnosis of digestive tract metastases from lung cancer, and especially for those patients with no clinical symptoms of digestive tract metastases from lung cancer, PET-CT can also make a definite diagnosis. Therefore, in the systemic assessment of patients with initially diagnosed primary lung cancer, clinicians should select the appropriate examination methods and pay attention to the assessment of abdominal hollow organs.

\section{Abbreviations}

CT: Computed tomography; PET-CT: positron emission tomography

\section{Declarations}

\section{Consent}

The patient died 3 years ago,so the Written informed consent was obtained from the patient'son for publication of this Case report and any accompanying images.

\section{Ethical approval}

Ethical approval from shaanxi provincial people's hospital ethics committee.A copy of the written consent is available for review by the Editor-in-Chief of this journal. 


\section{Competing interests}

The authors declare that they have no competing interests.

\section{Authors' contributions}

Shaochun Wang participated in treatment of the patient, contributed to collection of the clinical data and relevant literatures, and to writing of this manuscript.

Shuili Wang participated in treatment of the patient, and helped to edit the manuscript.

Wei Zhang and Yeqin Zhang participated in treatment of the patient, and revised and approved the manuscript. All authors read and approved the final manuscript.

\section{Data and Materials}

All the data and materials in the manuscript can be found in the patient' medical records, and data will be shared.

\section{Funding}

There was no funding.

\section{Acknowledgements}

The authors thank Dr. Zhou Zhongbin for his help in Journal recommendation and article submission. The authors also thank the support of the Natural Science

\section{References}

1. Parkin DM, Bray F, Ferlay J, et al. Global cancer statistics [J]. CA Cancer J Clin, 2005, 55(2):74-108.

2. Yang CJ, Huang JJ, Kang WY, et al. Gastro-intestinal metastasis of primary lung carcinoma: clinical presentations and outcome [J]. Lung Cancer, 2006, 54(3):319-323.

3. Hillers TK, Sauve MD, Guyatt GH. Analysis of published studies on the detection of extrathoracic metastases in patients presumed to have operable non-small cell lung cancer [J]. Thorax, 1994, 49(1):14-19.

4. Yoshimoto A, Kasahara K, Kawashima A. Gastrointestinal metastases from primary lung cancer [J]. Eur J Cancer, 2006, 42(18):3157-3160.

5. Rossi G, Marchioni A, Romagnani E, et al. Primary lung cancer presenting with gastrointestinal tract involvement: clinicopathologic and immunohistochemical features in a series of 18 consecutive cases [J]. J Thorac Oncol, 2007, 2(2):115-120.

6. Kim MS, Kook EH, Ahn SH, et al. Gastrointestinal metastasis of lung cancer with special emphasis on a long-term survivor after operation [J]. J Cancer Res Clin Oncol, 2009, 135 (2):297-301. 
7. Lee PC, Lo C, Lin MT, et al. Role of surgical intervention in managing gastrointestinal metastases from lung cancer[J]. World J Gastroenterology, 2011, 17(38):4314-4320.

8. Yaping Zhu, Weibing Lv, Bo Wang. One case of gastric metastasis from non small cell lung cancer[J]. Modern medicine and health care, 2017, 33(17): 2734-2735.

9. Kim MS, Kook EH, Ahn SH, et al. Gastrointestinal metastasis of lung cancer with special emphasis on a long-term survivor after operation [J]. J Cancer Res Clin Oncol, 2009, 135(2):297-301.

10. Sakai $\mathrm{H}$, Egi $\mathrm{H}$, Hinoi T, et al. Primary lung cancer presenting with metastasis to the colon: a case report [J]. World J Surg Oncol, 2012 10:127.

11. Wen Ji, Weiqing Chen, Banglun Liu. One case of gastric metastasis from Primary lung adenocarcinoma and literature review [J]. Journal of chongqing medical university, 2019, 44 (7).

12. Haendchen Bento L, Kazuyoshi Minata M, Pires Batista C, et al. Clinical and endoscopic aspects of metastases to the gastrointestinal tract [J]. Endoscopy, 2019, 51(7):646-652.

13. Kim MS, Kook EH, Ahn SH, et al. Gastrointestinal metastasis of lung cancer with special emphasis on a long-term survivor after operation [J]. J Cancer Res Clin Oncol, 2009, 135 (2): 297-301.

14. Stinchcombe TE, Socinski MA, Gangarosa LM, et al. Lung cancer presenting with a solitary colon metastasis detected on positron emission tomography scan [J]. J Clin Oncol, 2006, 24(30):49394940.

\section{Figures}



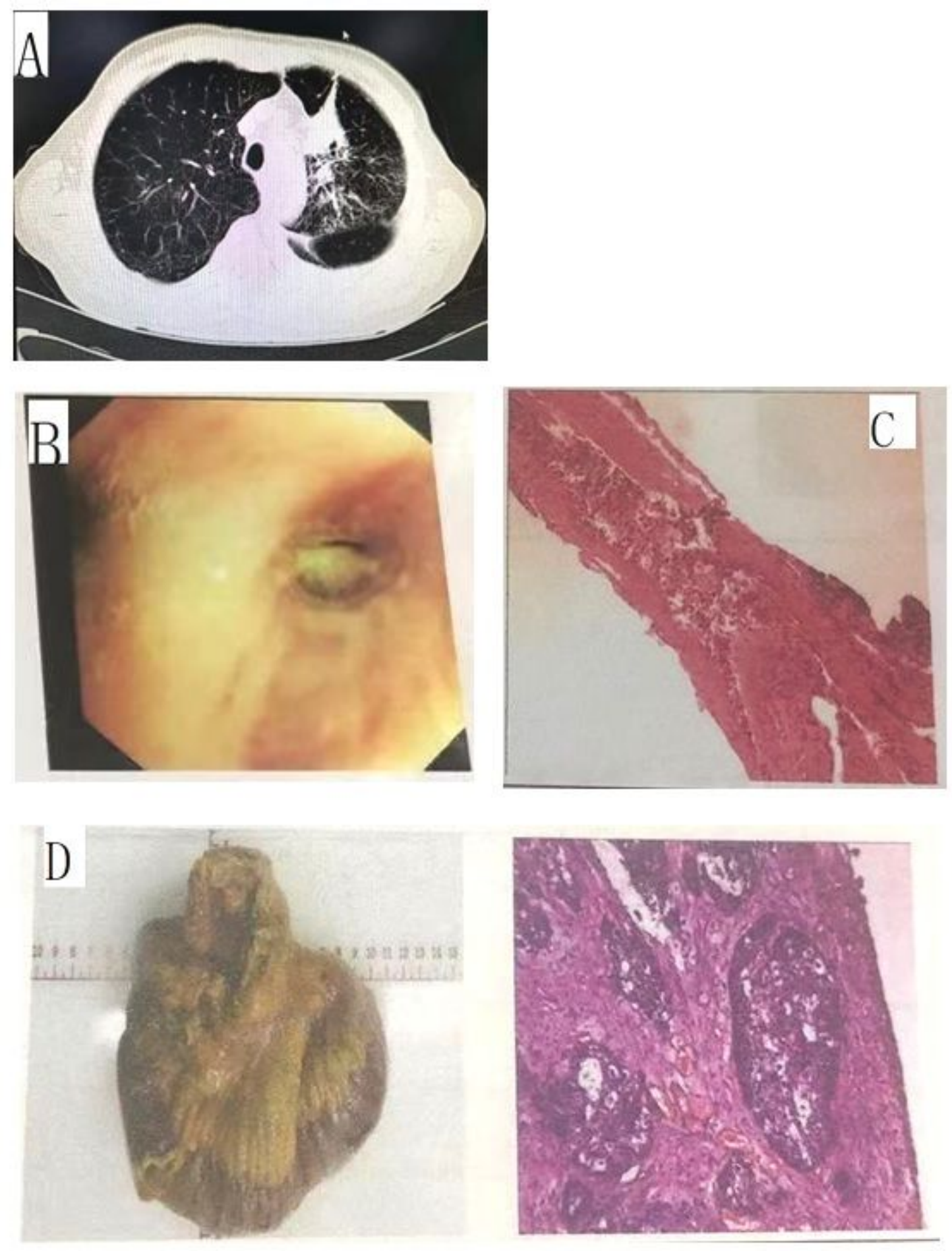

\section{Figure 1}

A: chest CT revealed a mass-like soft tissue density shadow in the left upper lobe (neoplasms cannot be excluded), left pleural thickening, and left pleural effusion. B: Bronchoscopy showed swelling and stenosis of the mucosa in the anterior segment of the left upper lobe, widening of the cristae, and a few nodular proliferations. C: Bronchoscopic biopsy pathology of the anterior segment of the left upper lobe showed moderately differentiated squamous cell carcinoma, immunohistochemistry: CK5/6 (+), P40 (+), p63 (+), CK7 (-), TTF-1 (-), NapsinA (-), and Ki-67 index 10\%. D: Postoperative pathology revealed poorly 
differentiated carcinoma of the small intestine, which was squamous-cell carcinoma, tumor invading the entire thickness of the small intestinal wall, tumor thrombus in the vessels. 